\title{
Microorganisms Profile and their Antimicrobial Resistance Pattern Isolated from the Lower Respiratory Tract of Mechanically Ventilated Patients in the Intensive Care Unit of A Tertiary Care Hospital in Dhaka
}

\author{
SARKER MOHAMMAD TAUHID JAMIL, ${ }^{1}$ MOHAMMAD OMAR FARUQ,${ }^{2}$ SHAFIQUS SALEHEEN, ${ }^{3}$ PRODIP KUMAR \\ BISWAS, ${ }^{4}$ MOHAMMED SHAHADAT HOSSAIN,${ }^{5}$ SYED ZAKIR HOSSAIN, ${ }^{4}$ MOHAMMAD ARIFUZZAMAN,${ }^{6}$ BIMAL \\ KUMAR BASU, ${ }^{7}$ ANWARUL KABIR, ${ }^{8}$ FERDOUS ALAM,${ }^{9}$ SURESH KUMAR TULSAN ${ }^{10}$
}

\begin{abstract}
:
Objective: Aim of the study was to know the microorganisms profile and determine the antibiotic resistance pattern of the LRT isolates from mechanically ventilated (MV) patients admitted to the ICU. This prospective observational study was done in Department of Critical Care Medicine (ICU) of BIRDEM General Hospital Dhaka from July, 20II to December, 201 I.

Methods: Blind Tracheal Aspirate or BronchoAlveolar Lavage or both from II 0 consecutive patients (total I 30 samples) admitted to the ICU requiring MV were cultured, identified, and antibiotic sensitivity was performed by standard methods.

Results: A total of I 30 samples were analyzed. Growth was obtained in $93.8 \%$ of the samples yielding I 43 organisms. Many (2I samples) yielded more than one organism. The major organism isolated were Acinetobacter sp. (54.5\%), Pseudomonas sp.(14.7\%), Klebsiella sp. (7.7\%). Candida sp. (7.0\%), Staphylococcus aureus(7.0\%), Escherichia coli (4.9\%).Proteus and Flavobacterium accounted for $4.2 \%$ of the isolates. All the isolates were highly resistant (>90\%) to cephalosporins and $>70 \%$ to fluoroquinolones. The frequency of third generation cephalosporin resistant E. coli, Klebsiellaand imipenem resistant Pseudomonas and Acinetobacter were $>90 \%$. Acinetobacter was remarkably resistant to most antibiotics including imipenem (>90\% resistant) and Piperacillin+Tazobactum (>85\% resistant), but most of the members of the Enterobacteriacae group and Acinetobacter showed maximum sensitivity to colistin (80\%-100\%).

Conclusion: Nonfermenters Gram Negative Bacilli (GNB)-Acinetobacter sp. \& Pseudomonas sp. are the most common etiological agents of LRT infections in ICU. There is an alarmingly high rate of resistance to cephalosporin and $\beta$-lactamase inhibitor group of drugs. Colistin was found to be the most sensitive drug against all GNB.
\end{abstract}

Keywords: Antimicrobial resistance, microorganism profile, intensive care unit, mechanically ventilated patients

1. Senior Medical officer, BIRDEM General Hospital. Dhaka.

2. Professor, Critical Care Medicine, BIRDEM General Hospital, Dhaka.

3. Medical Officer, OSD, DGHS, Dhaka.

4. Assistant Professor, Medicine, Dhaka Medical College, Dhaka.

5. Assistant Professor, Medicine, OSD, DGHS. Attached to Faridpur Medical College, Faridpur.

6. Assistant Professor, Neurology, Dhaka Medical College, Dhaka.

7. Senior Consultant, Pathology, Pabna General Hospital

8. Professor, Gastroenterology, BSMMU

9. Assistant Professor, Surgery, BSMMU

10. Assistant Professor, Department of Surgery, Kushtia Medical College

Correspondence: Dr. Sarker Mohammad Tauhid Jamil, Senior Medical officer, BIRDEM General Hospital, Shahbag, Dhaka, Mobile: no.01914389628,Email: tauhid36@gmail.com

\section{Introduction:}

Critically ill patients admitted in ICUs are always at a higher risk of developing nosocomial infections with resistant strains. ${ }^{1}$ Patients admitted in ICUs have an increased susceptibility to infection because of decreased mobility and increased use of invasive devices. ${ }^{2}$ LRTIs are the most common bacterial infections among patients in ICUs occurring in $10-25 \%$ of all ICU patients and resulting in high overall mortality, which may range from $22-71 \%$. 3,4 Most common bacterial agents of LRTI in the ICUs are Pseudomonas sp., Acinetobacter sp., Klebsiella sp., Citrobacter sp., Escherichia coli. ${ }^{5,6,7}$ In almost all cases, there is a need to initiate empirical antimicrobial treatment before obtaining the microbial culture results, but the situation is further complicated by the emergence of multiple beta lactamase producers and MDR pathogens. In a recent 
report, Infectious Disease Society of America specifically addressed three categories of GNB, namely ESBL producing Escherichia coli, and Klebsiella sp., MDR Pseudomonas sp., and carbapenem resistant Acinetobacter sp., as high priority bacterial pathogens. ${ }^{8}$ Infections with resistant strains of microorganisms in the ICUs lead to increased mortality and cost. ${ }^{9}$ All these major reports indicate the need for obtaining data on prevalent strains in the ICU along with the susceptibility pattern, to help in revising antibiotic policy and guiding clinicians for the better management of patients. Prevalent flora and antimicrobial resistance pattern may vary from region to region depending upon the predominant antibiotic use in that locality. Presently, data on pattern of organisms and their antibiotic susceptibility in ICUs of large hospitals of our country are lacking. Therefore, the present study is designed to know the microorganisms profile and determine the antimicrobial resistance pattern isolated from LRT of mechanically ventilated patients admitted to the ICU of BIRDEM General Hospital.

\section{Methods:}

It was aprospective observational study done in Department of Critical Care Medicine (ICU) of BIRDEM General Hospital (Tertiary Care Hospital) in Dhaka. July, 2011 to
December, 2011. Blind Tracheal Aspirate or Broncho Alveolar Lavage or both from 110 consecutive patients (total 130 samples) admitted to the ICU requiring MV were cultured, identified, and antibiotic sensitivity was performed by standard methods. SPSS software (Version-16) was used for calculation.

\section{Result:}

During the study period, a total of 130 samples were analyzed which included tracheal aspirate (110 samples) and broncho alveolar lavage (20 samples). Out of 130 samples growth was obtained from 122 samples (93.8\%) (Table 1)yielding 143 organisms.Out of 143 organisms, 123 (86.0\%) were GNB, 10(7.0\%) were Candida spp., and 10(7.0\%) were Gram positive cocci. In 21(16.1\%) samples yielded more than one organism.

Table-II shows the detail pattern of organisms isolated from lower respiratory tract specimen.

The commonest organism isolated from all samples was Acinetobacter sp.78 (54.5\%) followed by Pseudomonas sp. 21(14.7\%), Klebsiella sp.11 (7.7\%), Candida sp.10 (7.0\%), Staphylococcusaureus 10 (7.0\%), Escherichia coli 7(4.9\%).

Table-I

Sample profile and rate of positive culture from lower respiratory tract specimen.

\begin{tabular}{lccc}
\hline Samples & Total No. of sample & Samples yielding growth of organisms \\
& & N & $\%$ \\
\hline Tracheal aspirate & 110 & 102 & 92.7 \\
Broncho Alveolar Lavage(BAL) & 20 & 20 & 100 \\
\hline Total & 130 & 122 & 93.8 \\
\hline
\end{tabular}

Table-II

Pattern of organisms isolated from lower respiratory tract specimen

\begin{tabular}{|c|c|c|c|}
\hline Organism & Trahceal Aspirate (\%) & Broncho Alveolar Lavage (\%) & Total $(\%)$ \\
\hline \multicolumn{4}{|c|}{ Non fermenter organism } \\
\hline Acinetobacter sp. & 64 & 14 & $78(54.5)$ \\
\hline Pseudomonas sp. & 15 & 06 & $21(14.7)$ \\
\hline \multicolumn{4}{|l|}{ Enterobacteriaceae } \\
\hline Klebsiella sp. & 09 & 02 & $11(7.7)$ \\
\hline E.coli & 06 & 01 & $07(4.9)$ \\
\hline Proteus & 04 & 00 & $04(2.8)$ \\
\hline Flavobacterium & 02 & 00 & $02(1.4)$ \\
\hline \multicolumn{4}{|l|}{ Gram positive cocci } \\
\hline Staph aureus & 10 & 00 & $10(7.0)$ \\
\hline \multicolumn{4}{|l|}{ Fungus } \\
\hline Candida sp. & 09 & 01 & $10(7.0)$ \\
\hline Total & $119(83.2)$ & $24(16.8)$ & 143 \\
\hline
\end{tabular}

Note: Many samples yielded more than one organism. 
Table-III

The antibiotic resistance pattern of major organisms isolated from the lower respiratory tract of mechanically ventilated patients in intensive care unit.

\begin{tabular}{|c|c|c|c|c|}
\hline \multirow[t]{2}{*}{ Antibiotics } & \multicolumn{4}{|c|}{ Percent isolates showing antibiotic resistance } \\
\hline & $\begin{array}{c}\text { Acinetobacter sp } \\
\quad(n=78) * ¥\end{array}$ & $\begin{array}{c}\text { Pseudomonas } s p \\
(n=21) * ¥\end{array}$ & $\begin{array}{c}\text { Klebsiella sp } \\
\quad(n=11) * ¥\end{array}$ & $\begin{array}{c}\text { E.coli } \\
(n=07) * ¥\end{array}$ \\
\hline Colistin & $1 / 73(1.4 \%)$ & $2 / 12(16.7 \%)$ & $0 / 7(0 \%)$ & $0 / 4(0 \%)$ \\
\hline Piperacillin+Tazoba-ctum & $68 / 76(89.5 \%)$ & $4 / 18(22.2 \%)$ & $4 / 6(66.7 \%)$ & $3 / 6(50 \%)$ \\
\hline Imipenem & $73 / 78(93.6 \%)$ & $20 / 21(95.2 \%)$ & $9 / 11(81.8 \%)$ & $3 / 7(42.9 \%)$ \\
\hline Ceftriaxone & $78 / 78(100 \%)$ & $19 / 21(90.5 \%)$ & $10 / 11(90.9 \%)$ & $7 / 7(100 \%)$ \\
\hline Ceftazidime & $78 / 78(100 \%)$ & $19 / 21(90.5 \%)$ & $10 / 11(90.9 \%)$ & $7 / 7(100 \%)$ \\
\hline Cefotaxime & $78 / 78(100 \%)$ & $19 / 21(90.5 \%)$ & $11 / 11(100 \%)$ & $7 / 7(100 \%)$ \\
\hline Aztreonam & $76 / 78(97.4 \%)$ & $19 / 21(90.5 \%)$ & $11 / 11(100 \%)$ & $7 / 7(100 \%)$ \\
\hline Co-trimoxazole & $74 / 78(94.9 \%)$ & $17 / 78(80.9 \%)$ & $9 / 11(81.8 \%)$ & $4 / 7(57.1 \%)$ \\
\hline Amikacin & $78 / 78(100 \%)$ & $16 / 21(76.2 \%)$ & $9 / 11(81.8 \%)$ & $4 / 7(57.1 \%)$ \\
\hline Netilmicin & $63 / 78(80.8 \%)$ & $16 / 21(76.2 \%)$ & $9 / 11(81.8 \%)$ & $4 / 7(57.1 \%)$ \\
\hline Gentamicin & $76 / 78(97.4 \%)$ & $20 / 21(95.2 \%)$ & $10 / 11(90.9 \%)$ & $6 / 7(85.7 \%)$ \\
\hline Ciprofloxacin & $76 / 78(97.4 \%)$ & $15 / 21(71.4 \%)$ & $10 / 11(90.9 \%)$ & $7 / 7(100 \%)$ \\
\hline
\end{tabular}

*resistance to each antibiotic is indicated as $\mathrm{X} / \mathrm{Y}$; where $\mathrm{X}=$ number of isolates resistance to particular antibiotic, \& $\mathrm{Y}=$ total number of isolates for which antibiotic susceptibility was tested.

$¥=$ percentage of resistance is given within brackets; $n=$ total isolates

The antibiograms of common isolates are shown in Table 3. Majority of the isolates were highly resistant ( $>90 \%)$ to $3^{\text {rd }}$ generation cephalosporins and $>70 \%$ to ciprofloxacin. The frequency of $3^{\text {rd }}$ generation cephalosporin resistant $E$. coli, Klebsiellaand imipenem resistant Pseudomonas and Acinetobacter were $>90 \%$. Acinetobacter was remarkably resistant to most antibiotics including imipenem ( $>90 \%$ resistant) and Piperacillin+Tazobactum ( $>85 \%$ resistant), but most of the members of the Enterobacteriacaegroup and Acinetobacter showed maximum sensitivity to colistin (83\%-100\%). Resistance pattern of organism to aminoglycosides was variable ranging from $57.1 \%$ to $100 \%$. About $50 \%$ of isolated S. aureus were methicillin resistant (MRSA). No sensitivity was done for Candida $s p$

\section{Discussion:}

Our study included types and antibiotic susceptibility pattern of organisms isolated from the lower respiratory tract of mechanically ventilated patients in the intensive care unit, but it did not attempt to investigate the underlying disease condition of patients or their sources of infection.

In this study, growth was obtained from $93.8 \%$ samples (T/ A, BAL) which probably were due to the fact that most patients either had prior respiratory problems or were in ventilators.

Gram negative bacteria were isolated at a significantly higher rate $(86 \%)$ than gram positive bacteria and Candida. Acinetobacter, Pseudomonas, Klebsiella, E.coliand Candida were the most prevalent pathogens recovered from our ICU patients (Table-II). The predominant bacterial isolates reported in the Jordanian, Indian and European studies were almost similar to our results..$^{9,10,11,12}$

Reduction in antimicrobial resistance in the ICUs has been a goal for all intensive care units as it improves the outcome and reduces total expenses as well as duration of ICU stay. The extreme antibiotic use results in the emergence of MDR microorganisms in the ICU environment. The present study revealed high prevalence of antibiotic resistant organisms in our ICU. More than 90\% Pseudomonas sp. showed resistance to $3^{\text {rd }}$ generation cephalosporins and $>70 \%$ resistance to fluoroquinolons. In $2005 \&$ 2006-2007, two study conducted in the same ICU reported $>80 \%$ of Pseudomonas was resistant to $3^{\text {rd }}$ generation cephalosporins. ${ }^{13,14}$ But it has been observed that the frequency of fluoroquinolon and imipenem resistant 
Pseudomonas (71.4\% and 95.2\%) has increased in the present study compared to that of 2005 (48\% and $36 \%$ respectively). In present study, colistin was the most effective drug ( $>80 \%$ sensitive) against pseudomonas followed by piperacillin+tazobactum combination ( $>75 \%$ sensitive).

In present study, Acinetobactersp was the commonest $(54.5 \%)$ organism isolated from LRT specimen but in the study conducted in the same ICU in 2006-2007 Acinetobacterwas isolated only $27.5 \% .{ }^{14}$ Though this is commonly isolated from skin and throat of healthy people, it is also known to colonize respirators, respiratory tubing and intravenous catheters in ICU causing serious and often fatal opportunistic infections. They are generally resistant to most classes of antimicrobials and emergence of imipenem resistant strains in many parts of the world is alarming and a threat to the effective management of these infections. ${ }^{15} \mathrm{In}$ our study, Acinetobacterwas remarkably resistant to most antibiotics including imipenem (93.6\% resistance) and piperacillin+tazobactum $(89.5 \%$ resistance $)$ but Acinetobacterand most of the members of the Enterobacteriacaegroup like E.coli, Klebsiella were maximally susceptible to colistin ( $>98 \%$ sensitive). The susceptibility of these bacteria to cephalosporins was uniformly poor in our study ( $>90 \%$ resistant). This was probably due to over use of cephalosporins in the indoor and ICU patients. Resistance pattern of these organisms to aminoglycosides showed marked variability ranging from $57.1 \%$ to $100 \%$.

Candida species was the fourth frequently isolated organism in our ICU. Both C. albican and non- albican Candida species were found. High number isolation of Candida might be due to the presence of underlying conditions like poor nutritional status, diabetes mellitus and the use of steroids and broad spectrum antibiotics.

\section{Conclusion}

We conclude that GNB are the most common etiological agents of LRTIs in our ICU. Acinetobacter\& Pseudomonas species hold the top of the list. There is an alarmingly majority of the isolates showed high resistance to cephalosporins, aminoglycosides \& quinolones. Compared to previous two studies, overall resistance pattern of Pseudomonas \& Acinetobacter to carbapenem became worse. Colistin was found to be the most sensitive drug against all GNB. The findings of this study might help clinicians to formulate their first line empirical antibiotic treatment regimens for the patients admitted in ICUs and judicious use of antimicrobial agents is essential to prevent the emergence of MDR bacteria in the ICUs.

\section{Conflict of Interest : None}

\section{References:}

1. Singh AK, SenMR, Anupurba S, Bhattacharya P. Antibiotic sensitivity pattern of the bacteria isolated from nosocomial infections in ICU. Journal ofCommunicable Diseases 2002; 34:257-263.

2. Rice LB. Controlling antibiotic resistance in the ICU: Different bacteria, different strategies. Cleveland ClinicJournal of Medicine 2003;70:793-800.

3. Vincent JL, Bihari DJ, Suter PM, Bruining HA, White J, Nicolas-Chanoin MH, etal. The prevalence of nosocomial infection in intensive care units in Europe. JAMA 1995; 274:639-44.

4. Chastre J, Fagon JY. Ventilator-associated pneumonia. Am J RespirCrit Care M ed 2002;165:867-903.

5. Navaneeth BV, Belwadi MR. Antibiotic resistance among gram-negative bacteria of lower respiratory tract secretion in hospitalized patients. Indian J Chest Dis Allied Sci 2002; 44:173-

6. Gonlugur U, Bakici MZ, Akkurt I, Efeoglu T. Antibiotic susceptibility patterns among respiratory isolates of Gram negative bacilli in Turkish University Hospital. BMC Microbiology 2004;4:32-4.

7. Mukhopadhyay C, Bhargava A, Ayyagari A. Role of mechanical ventilation and development of multidrug resistant organisms in hospital acquired pneumonia. Indian J Med Res 2003;118:229-35.

8. Talbot GH, Bradley J, Edwards JE Jr, Gilbert D, Scheld M, Bartlett JG. Bad bugs need drugs: An update on the Development pipeline from the antimicrobial availability Task Force of the Infectious Diseases Society of America. Clin Infect Dis 2006;42:657-68 [erratum:2006;42:1065 9.Kaul S, BahmadathanKN, Jagannati M, Sudarsanam TD, Pitchamuthe K, Abraham OC et al. One year trends in the gram negative bacterial antibiotic susceptibility patterns in a medical intensive care unit in South India. Indian J Med Microbiology 2007;25:230-5.

10. Shehabi AA, Baadran I. Microbial infection and antbiotic resistance patterns among Jordanian intensive care patients. Eastern Mediterranean Health Journal 1996;2:515-520.

11. Patwardhan RB, Dhakephalkar PK, Niphadkar KB, Chopade BA. A study on nosocomial pathogens in ICU with special reference to multiresistant Acinetobacter baumannii harbouring multiple plasmids. Indian J Med Res 2008;128: 178-187.

12. Verbist L. Epidemiology and sensitivity of 8625 ICU hematology/ oncology bacterial isolates in Europe. Scand J Infectious Disease 1993; Supplementum 91:14-24.

13. Basunia MRA, Rahman MR, Faruq MO, Huq F, Ahsan A, Hasan R, Ahmed B. Microbial pathogens and antibiotic sensitivity at intensive care unit of BIRDEM: A retrospective study. Bangladesh JMedicine 2005;16:14-20.

14. Barai et al. Bacterial profile \& antimicrobial resistance pattern in an intensive care unit of a tertiary care hospital in Dhaka. Ibrahim Medical College Journal 2010; 4(2):66-69.

15. Mulvey MR, Simor AE. Antimicrobial resistance in hospitals: How concerned should we be? CanadianMed Assoc J 2009;180:408-415. 